Supporting Information

\title{
Stable Cycling of Sodium Metal All-Solid-State Batteries with Polycarbonate-Based Polymer Electrolytes
}

Christofer Sångeland, Ronnie Mogensen, Daniel Brandell, Jonas Mindemark*

Department of Chemistry - Ångström Laboratory, Uppsala University, Box 538, SE-751 21

Uppsala, Sweden

* Corresponding author. E-mail: jonas.mindemark@kemi.uu.se 


\section{Prussian blue cathode material characterization}

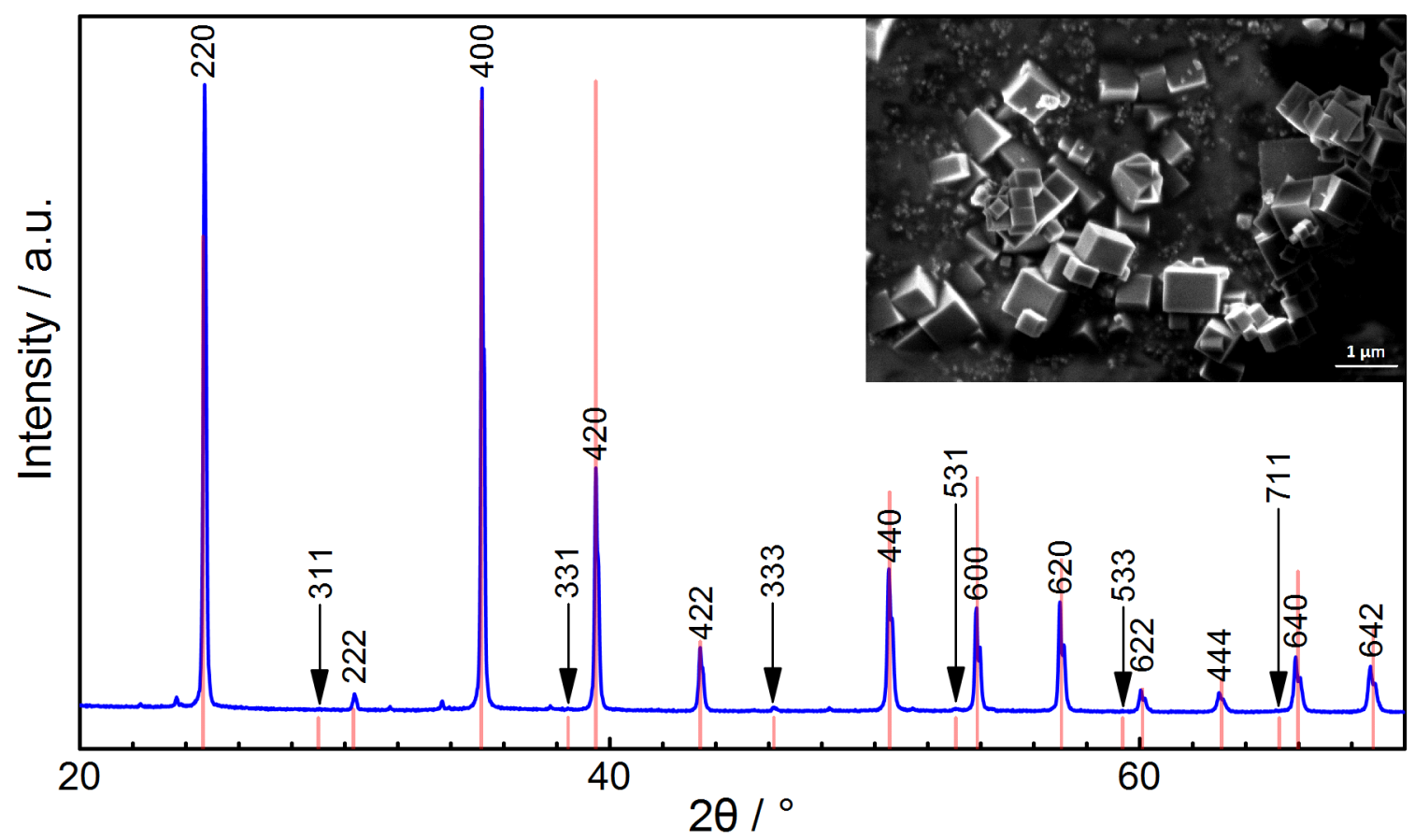

Figure S1. X-ray diffractogram of synthesized cathode active material. Red lines indicate peak positions of cubic "Berlin green" or $\mathrm{Fe}_{2}(\mathrm{CN})_{6}$ according to Widmann et al. ${ }^{1}$ Berlin green shares the same unit cell as Prussian blue (space group Fm-3m), but slightly smaller. The inset shows an SEM micrograph of cubic Prussian blue crystals. XRD data was obtained using a Bruker TwinTwin instrument with a $\mathrm{CuK} \alpha$ radiation source and a Lynxeye XE PSD detector. SEM imaging was done using a LEO 1550 Scanning Electron Microscope. 


\section{VFT fitting parameters}

The Vogel-Fulcher-Tammann (VFT) equation describes the relationship between ionic conductivity and temperature in amorphous polymer electrolytes:

$$
\sigma=\frac{A}{\sqrt{T}} e^{-\frac{B}{T-T_{0}}}
$$

Conductivity data was fitted to Equation S1 using non-linear curve fitting with a LevenbergMarquardt iteration algorithm. $T_{0}$ was initially fixed at $200 \mathrm{~K}$ until the fit converged; after this, all parameters including $T_{0}$ were set free until final convergence.

Table S1. Values obtained for parameters in the fitted VFT equation. $n$ refers to the carbonate:NaFSI ratio.

\begin{tabular}{cccccc}
\hline$n$ & $\begin{array}{c}\text { NaFSI weight } \\
\text { concentration } \\
{[\text { wt\% }]}\end{array}$ & $\begin{array}{c}\text { NaFSI molar } \\
\text { concentration } \\
{[\text { mol\%] }}\end{array}$ & $\begin{array}{c}A \\
{\left[\mathrm{~S} \mathrm{~cm}^{-1} \mathrm{~K}^{1 / 2}\right]}\end{array}$ & $\begin{array}{c}B \\
{[\mathrm{~K}]}\end{array}$ & $\begin{array}{c}T_{0} \\
{[\mathrm{~K}]}\end{array}$ \\
\hline 1 & 67 & 50.0 & 0.0636 & 113 & 271 \\
2 & 50 & 33.3 & 2.14 & 888 & 227 \\
3 & 40 & 25.0 & 3.19 & 1140 & 230 \\
5 & 29 & 16.7 & 4.66 & 1270 & 223 \\
8 & 20 & 11.1 & 2.90 & 1380 & 214 \\
13 & 13 & 7.1 & 0.246 & 998 & 226 \\
21 & 8.7 & 4.5 & 0.0877 & 1040 & 224 \\
\hline
\end{tabular}




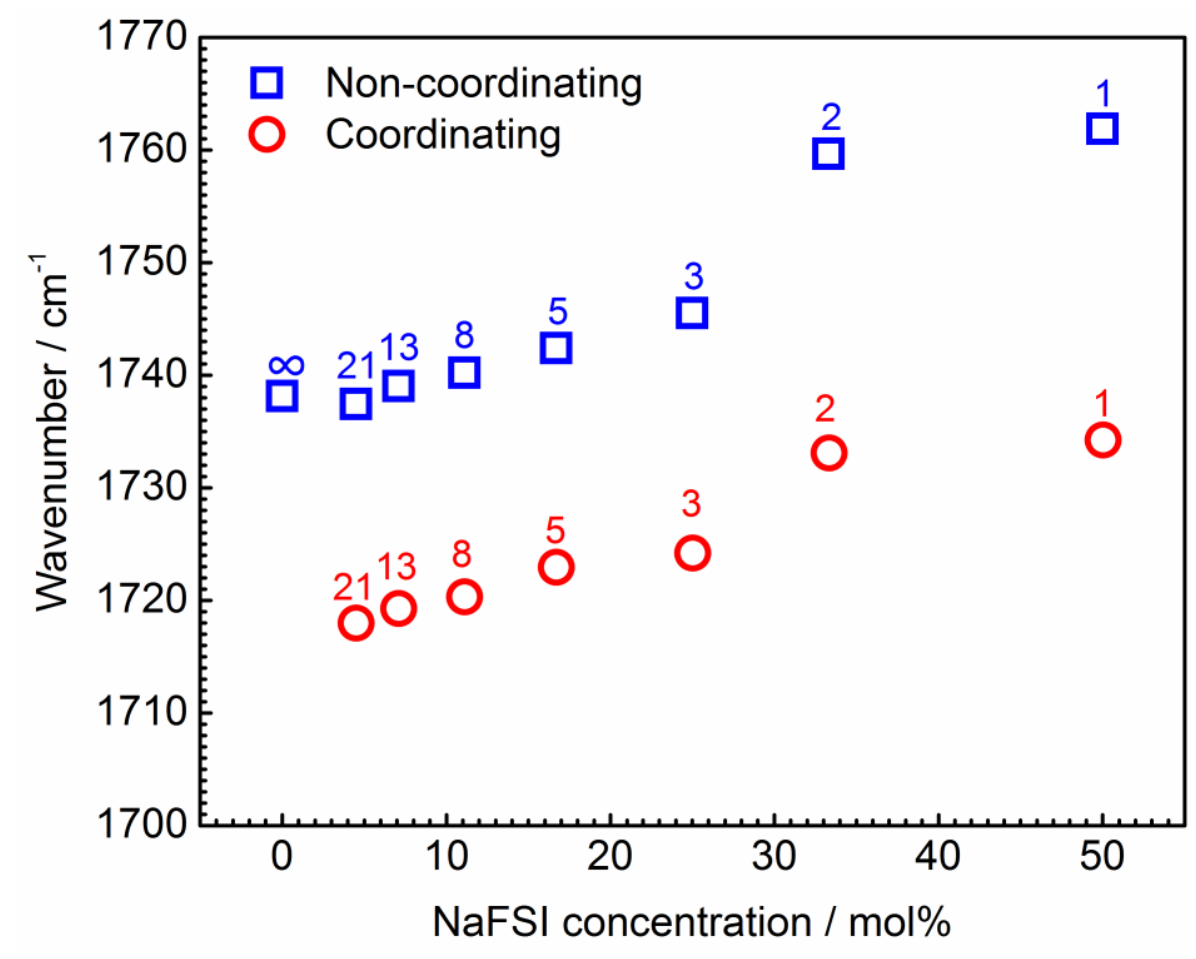

Figure S2. Variation in FTIR peak position with NaFSI concentration for coordinating and noncoordinating PTMC carbonyl groups. The annotated numbers refer to the carbonate: $\mathrm{Na}^{+}$ratio $n$ with $\infty$ corresponding to PTMC without added salt.

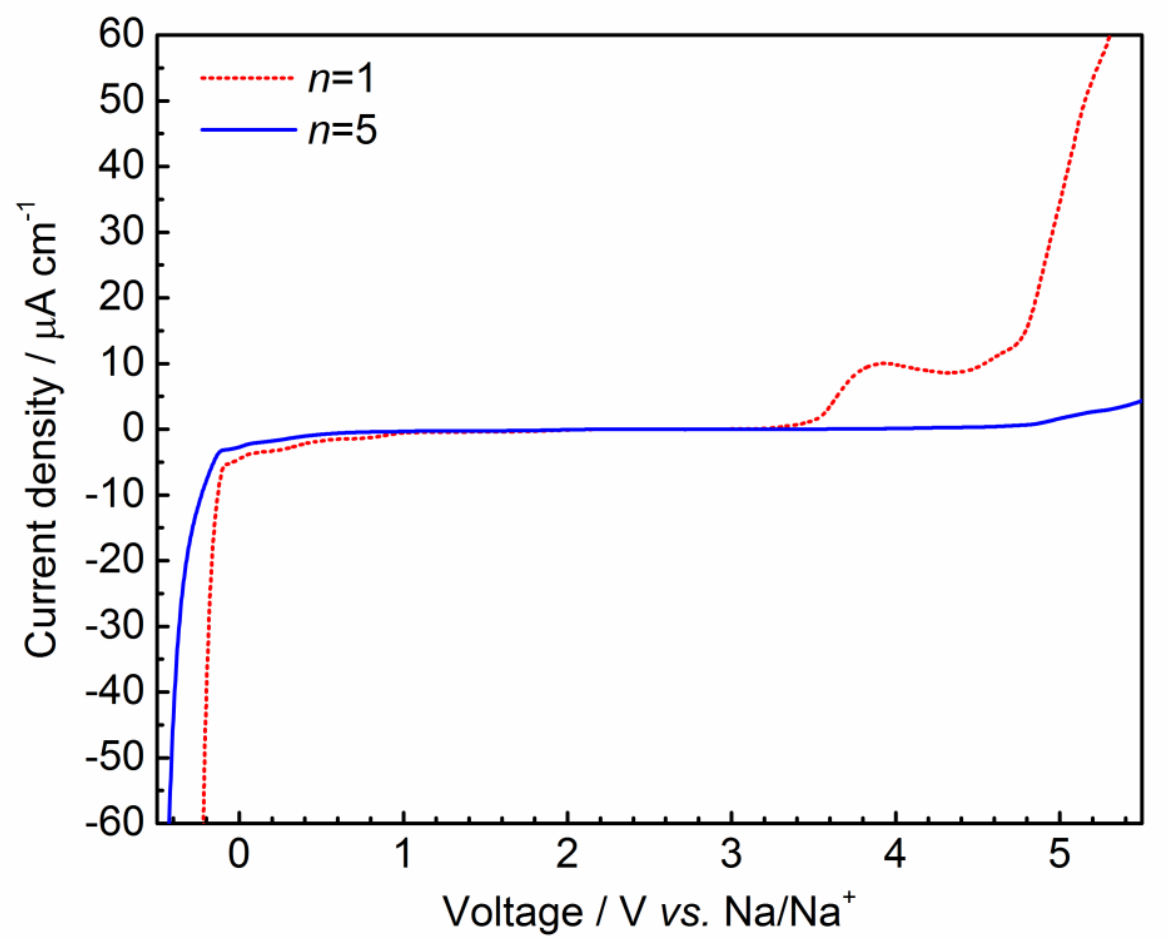

Figure S3. Linear sweep voltammetry of PTMC 5 NaFSI (blue solid line) and PTMC ${ }_{1} \mathrm{NaFSI}$ (red dashed line) at $60{ }^{\circ} \mathrm{C}$. 


\section{References}

(1) Widmann, A.; Kahlert, H.; Petrovic-Prelevic, I.; Wulff, H.; Yakhmi, J. V.; Bagkar, N.; Scholz, F. Structure, Insertion Electrochemistry, and Magnetic Properties of a New Type of Substitutional Solid Solutions of Copper, Nickel, and Iron Hexacyanoferrates/Hexacyanocobaltates. Inorg. Chem. 2002, 41, 5706-5715. 